\title{
A Simulation Study of Recessed Type Hydrostatic Journal Bearing
}

\author{
Shih-Ming Wong, Ta-Hua Lai, Min-Cheng Lin, and Shih-Chieh Lin \\ Department of Power Mechanical Engineering, National Tsing-Hua University, Hsinchu 300, Taiwan
}

\begin{abstract}
In this paper, a simulation model is established to study effects of the bearing system parameters on bearing performance. The static performance (such as load capacity, stiffness and flow) about various dimensions of multi-recess type hydrostatic journal bearings with constant-resistant restrictor compensation was investigated. The parameters studied includes length/diameter ratio, number of recess, recess length ratio, recess width ratio, initial ratio between recess pressure to supply pressure, and eccentricity.
\end{abstract}

\section{Introduction}

Hydrostatic bearing has been characterized with high damping, low friction and low wear. For high precision machining, hydrostatic bearing is widely adopted in recent years.

Many researchers had been devoted to the study of hydrostatic bearing systems. Dowson [1] studied the effects of the depth of recess on pressure distribution over the recess area. O'Donoghue [2] adopted the finite difference method to study the distribution of pressure over the bearing area. Majumdar [3] studied the relationship between the restrictor and load capacity. Gosh [4-6] studied the loading capacity and flow rate for multi-pad bearing. Row [7] studied the effects of pocket design on torque. Ho [8] conducted experiments to confirm simulations results. Liang [9] studied the hydrodynamics effects. Donoghue [10], Ghigliazza [11] and Elsherbiny [12] proposed ways for hydrostatic bearing system design.

In this study, a simulation model is established to study effects of the bearing system parameters on bearing performance. The static performance (such as load capacity, stiffness and flow) about various dimensions of multi-recess type hydrostatic journal bearings with constant-resistant restrictor compensation was investigated. The parameters studied includes length/diameter ratio, number of recess, recess length ratio, recess width ratio, initial ratio between recess pressure to supply pressure, and eccentricity.

In the following section, the fundamental theories for the simulation models are briefly reviewed. A series of simulations are then conducted to studied effects of parameters on system performances. Finally, conclusions are made based on the results.

\section{Simulation model}

The recess dimension parameters of hydrostatic journal bearings, as shown in Fig. 1, includes bearing diameter D, bearing length $\mathrm{L}$, land width $\mathrm{a}$, and arc length of recesses b (related to recess size).

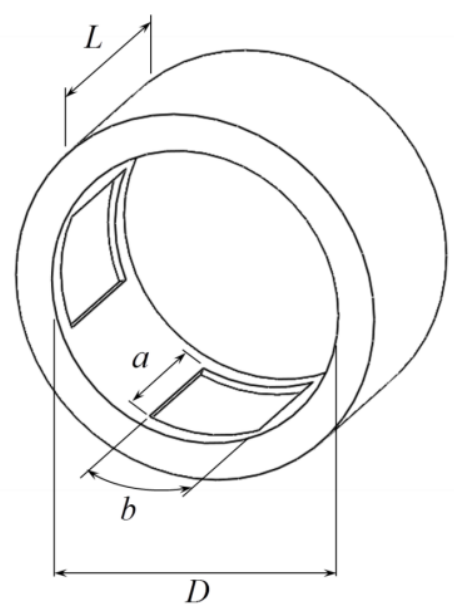

Figure 1. Journal bearing geometry parameters.

To obtain the pressure distribution of the oil film in the hydrostatic journal bearing, the generalized 2dimensional Reynolds equation was used ${ }^{1,2,3)}$ (neglecting the time dependent terms).

$$
\frac{\partial}{\partial x}\left(\frac{\rho h^{3}}{12 \mu} \frac{\partial p}{\partial x}\right)+\frac{\partial}{\partial y}\left(\frac{\rho h^{3}}{12 \mu} \frac{\partial p}{\partial y}\right)=0
$$

where $\mu$ is coefficient of absolute viscosity, and $h$ is the film thickness.

The bearing surface is unwrapped in the finite difference form, represented in the $x-y$ plane, as shown in Fig. 2. The pressure at any point $(x, y)$ was solved iteratively using Finite Different Method (FDM), which satisfy the following boundary conditions: 


$$
\begin{aligned}
& p(\theta, \pm 1)=0 \\
& p=p_{r} \text { at the } r \text { th reces } \\
& p(\theta, y)=p(\theta+2 \pi, y) \text { for periodicity }
\end{aligned}
$$

where $\theta$ is angular coordinate, $p_{r}$ is recess pressure at $r$ th recess.
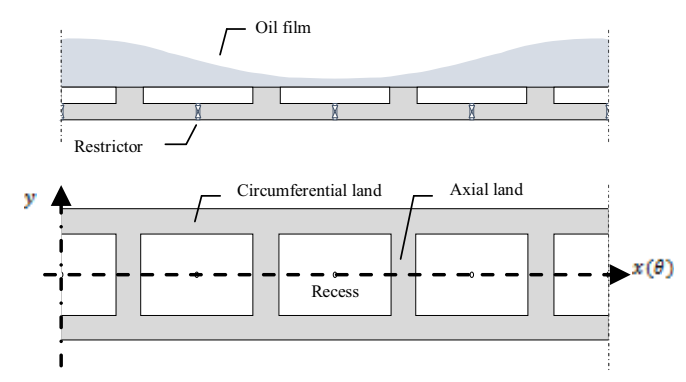

Figure 2. Hydrostatic bearing surface unwrapped.

The solution satisfying boundary conditions yields the pressure distribution of the oil film, which leads to the determination of load capacity, stiffness, and the power consumption of the bearing.

\section{Simulation conditions, results and discussions}

In order to study effects of the bearing system parameters on bearing performance, a series of simulations had been studied. The system parameters and levels are listed in table 1 . The performance of the bearing system includes dimensionless loading capacity, $\bar{W}=w /\left(P_{s} L D\right)$ stiffness,

\begin{tabular}{|c|c|c|}
\hline Parameters & Symbols & Levels \\
\hline $\begin{array}{l}\text { length/diameter } \\
\text { ratio }\end{array}$ & $\mathrm{L} / \mathrm{D}$ & $0.5 、 1.0 、 1.5$ \\
\hline $\begin{array}{c}\text { Recess length } \\
\text { ratio } \\
\end{array}$ & $\mathrm{a} / \mathrm{L}$ & $0.25 、 0.50 、 0.75$ \\
\hline $\begin{array}{c}\text { Recess width } \\
\text { ratio }\end{array}$ & $\mathrm{Nb} /(\pi \mathrm{D})$ & $0.25 、 0.50 \cdot 0.75$ \\
\hline $\begin{array}{c}\text { Number of } \\
\text { recess }\end{array}$ & $\mathrm{N}$ & $4 \cdot 5 \cdot 6 \cdot 7$ \\
\hline $\begin{array}{c}\text { Eccentricity } \\
\text { ratio } \\
\end{array}$ & $\varepsilon$ & $0.1 \cdot 0.3 \cdot \ldots \cdot 0.9$ \\
\hline $\begin{array}{c}\text { Initial pressue } \\
\text { ratio }\end{array}$ & $\beta_{0}$ & $0 、 0.1 、 \cdots 、 1.0$ \\
\hline Attitude angle & $\phi$ & $0, \pi / \mathrm{N}$ \\
\hline
\end{tabular}
$\bar{K}=k h_{0} /\left(P_{s} L D\right)$ and flow rate, $\bar{Q}=q \eta /\left(P_{s} h_{0}^{3}\right)$.

Table 1. System parameters and levels for simulation tests.

\subsection{Dimensionless loading capacity}

The simulation results show that the effects of initial pressure ratio between the recess pressure and the supplied pressure on the loading is the most significant, and then the eccentricity ratio, the recess length ratio, length/diameter ratio, number of recess, recess width ratio and attitude angle.

Fig. 3 shows the interaction effects of the initial pressure ratio and the eccentricity ratio on loading capacity. It was observed that a maximum loading can be derived when the initial pressure ratio is around 0.5. And the loading capacity is generally increased as an increase in eccentricity ratio. In other words, the increase in load will increase the eccentricity

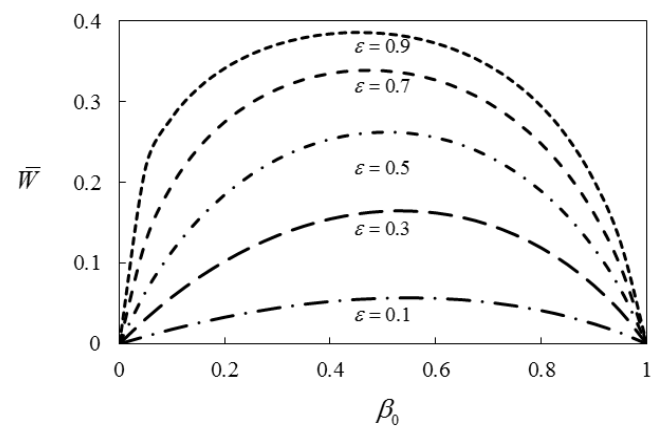

Figure 3. Interaction effects of the pressure ratio and the eccentricity ratio on loading capacity.

Fig. 4 shows the interaction effects of the recess length ratio and the length/diameter ratio on loading capacity. It was observed that the loading increased as the recess length ratio increased. And the loading capacity is generally increased as a decrease in length/diameter ratio. However, the decrease in length will decrease the load capacity.

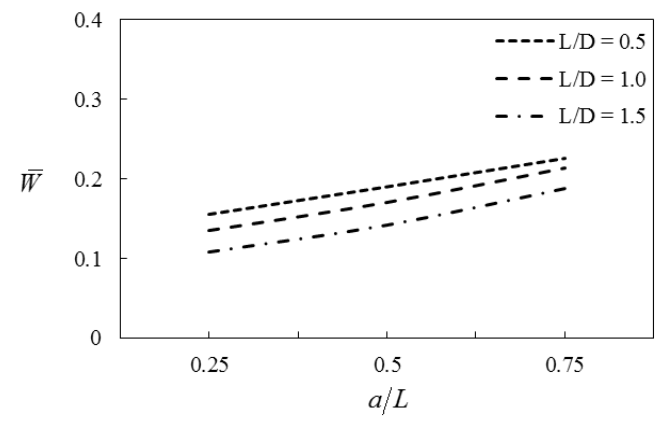

Figure 4. Interaction effects of the recess length ratio and the length/diameter ratio on loading capacity.

Fig. 5 shows the interaction effects of the recess width ratio and the number of recess on loading capacity. It was observed that the loading capacity is slightly increased as the number of recess is increased. And the effects of recess width ratio on the loading capacity is not so significant. However, a higher loading can be achieved with a ratio of 0.5 .

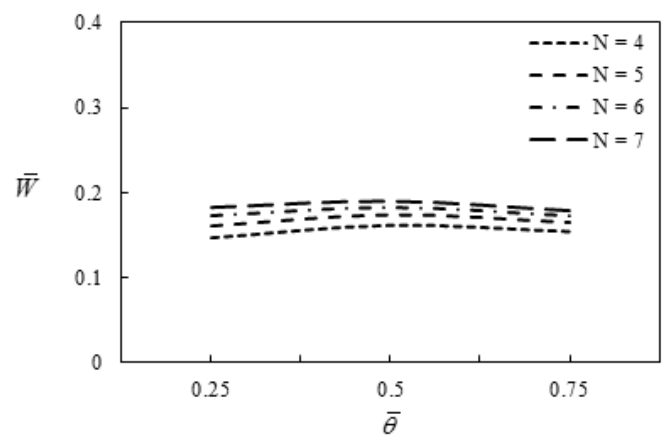

Figure 5. Interaction effects of the recess width ratio and the number of recess on loading capacity. 


\subsection{Dimensionless stiffness}

The simulation results show that the effects of initial pressure ratio between the recess pressure and the supplied pressure on the stiffness is the most significant, and then the eccentricity ratio, the recess length ratio, length/diameter ratio, number of recess, recess width ratio and attitude angle.

Fig. 6 shows the interaction effects of the initial pressure ratio and the eccentricity ratio on stiffness. A strong interaction effect of the initial pressure ratio and the eccentricity ratio on stiffness can be observed. When the initial pressure ratio is larger than 0.5 , the increase of eccentricity ratio will results in a decrease in stiffness. The dimensionless stiffness for a pressure ratio of 0.5 with no eccentricity is around 0.55 while that is reduced to 0.25 with an eccentricity of 0.8 .

When the initial pressure ratio is smaller than 0.4 , the increase of eccentricity ratio will results in an increase in stiffness in the beginning and a decrease in stiffness with the further increase in the eccentricity ratio. In other words, when the initial pressure is low, the increase in loading will result in an increase in stiffness in the beginning and then a decrease in stiffness if the loading is further increased.

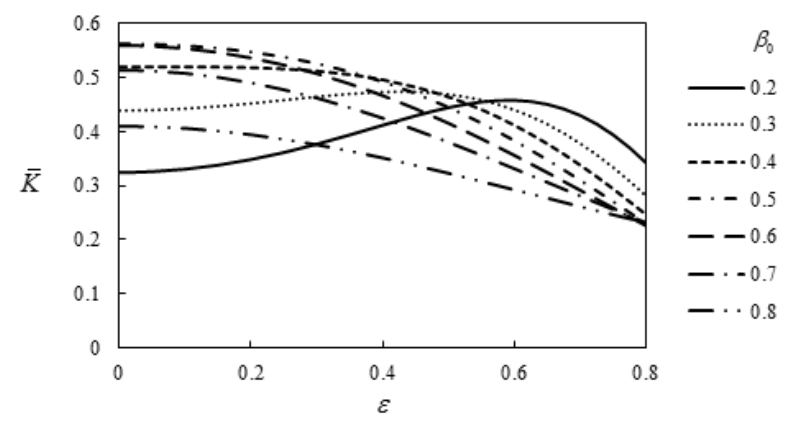

Figure 6. Interaction effects of the pressure ratio and the eccentricity ratio on stiffness.

Fig. 7 shows the interaction effects of the recess length ratio and the length/diameter ratio on stiffness. It was observed that the stiffness increased as the recess length ratio increased. And the stiffness is decreased as the length/diameter ratio increased.

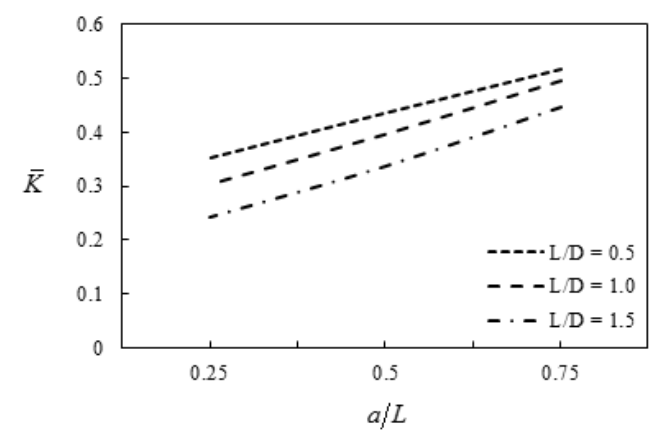

Figure 7. Interaction effects of the recess length ratio and the length/diameter ratio on stiffness.

Fig. 8 shows the interaction effects of the recess width ratio and the number of recess on stiffness. It was observed that thestiffness is slightly increased as the number of recess is increased. And the effects of recess width ratio on the stiffness is not so significant. However, a higher stiffness can be achieved with a ratio of 0.5 .

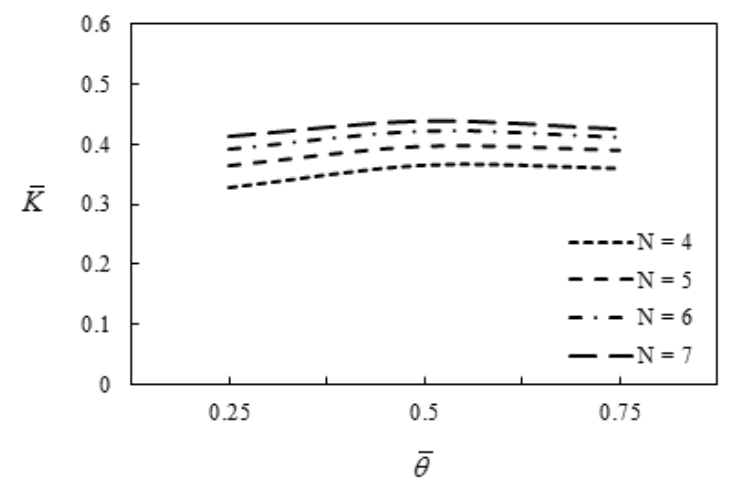

Figure 8. Interaction effects of the recess width ratio and the number of recess on Stiffness.

\subsection{Dimensionless flow rate}

The simulation results show that the effects of initial pressure ratio between the recess pressure and the supplied pressure on the flow rate is the most significant again, and then the recess length ratio, length/diameter ratio, recess width ratio, number of recess, the eccentricity ratio, and attitude angle.

Fig. 9 shows the interaction effects of the initial pressure ratio and the recess length ratio on flow rate. It was observed the flow rate increased as the initial pressure ratio or the recess length ratio increased. The increase in pressure ratio is accompanying with a lower flow resistance of the restrictor in simulated cases. And a larger recess length ratio also means that the land area of the bearing is thinner and a lower flower resistance for the oil to flow out.

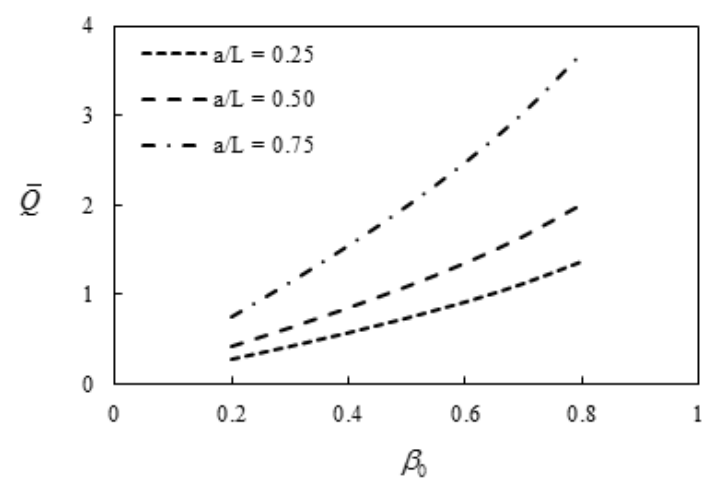

Figure 9. Interaction effects of the pressure ratio and the eccentricity ratio on stiffness.

Fig. 10 shows the interaction effects of the recess width ratio and the length/diameter ratio on flow rate. It was observed that the flow rate decreased as the length/diameter ratio increased. This is so since the width of land will increase as the length/diameter ratio increased, and hence the flow resistance will also increase as the length/diameter increased. And the flow rate is slightly increased as the increase in recess width ratio. This is so since the pocket area is increased and the land area is reduced as the recess width increased. The decrease in the land area generally results in a lower flow resistance. 


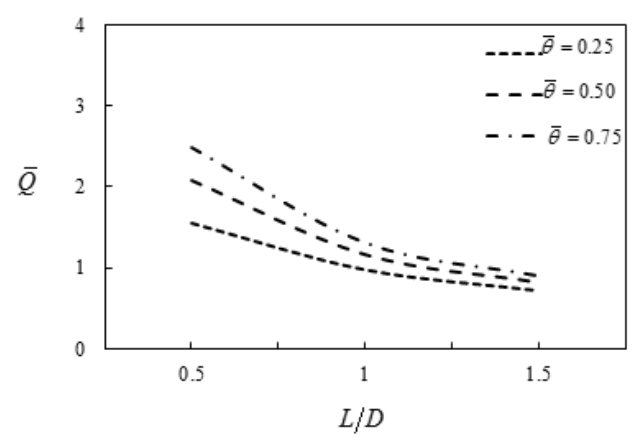

Figure 10. Interaction effects of the recess width ratio and the length/diameter ratio on stiffness.

Fig. 11 shows the interaction effects of the eccentricity ratio and the number of recess on flow rate. It was observed that the flow rate is slightly increased as the number of recess is increased. And the flow rate is also slightly increased as the eccentricity rate is increased.

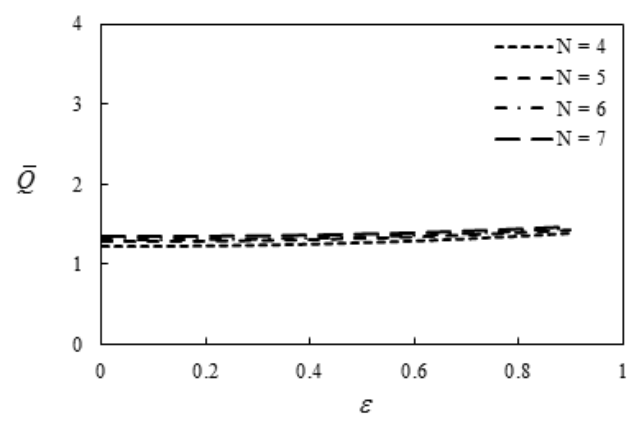

Figure 11. Interaction effects of the eccentricity ratio and the number of recess on Stiffness.

\section{Conclusions}

In this paper, a simulation model is established to study effects of the bearing system parameters on bearing performance. The static performance (such as load capacity, stiffness and flow) about various dimensions of multi-recess type hydrostatic journal bearings with constant-resistant restrictor compensation was investigated.
The simulation results show that the effects of initial pressure ratio between the recess pressure and the supplied pressure on all three performance index is the most significant. It was observed that a maximum loading can be derived when the initial pressure ratio is around 0.5 .

A strong interaction effect of the initial pressure ratio and the eccentricity ratio on stiffness can be observed. When the initial pressure ratio is larger than 0.5 , the increase of eccentricity ratio will results in a decrease in stiffness. The dimensionless stiffness for a pressure ratio of 0.5 with no eccentricity is around 0.55 while that is reduced to 0.25 with an eccentricity of 0.8 .

It was observed the flow rate increased as the initial pressure ratio or the recess length ratio increased. The increase in pressure ratio is accompanying with a lower flow resistance of the restrictor in simulated cases. And a larger recess length ratio also means that the land area of the bearing is thinner and a lower flower resistance for the oil to flow out.

\section{References}

1. D. Dowson and C. Taylor, ASME, 83, 2 (1960)

2. J. O'Donoghue and W. Rowe, Tribology, 1, 4 (1968)

3. B. Majumdar, Wear, 14, 6, (1969)

4. B. Ghosh, Wear, 21, 2 (1972)

5. B. Ghosh, Wear, 23, 3 (1973)

6. M. Ghosh and B. Majumdar, Tribology International, 13, 2 (1980)

7. W. Rowe and D. Koshal, Wear, 43, 1 (1977)

8. Y. HO and N. Chen, Wear, 52, 2 (1979)

9. P. Liang, C. Lu, J. Ding and S. Chen, Tribology International, 67 (2013)

10. J. O'Donoghue, W. Rowe and C. Hooke, Wear, 14, 5 (1969)

11. R. Ghigliazza, R. Michelini and L. Rossi, Tribology, 3, 3 (1970)

12. M. El-Sherbiny, F. Salem and N. El-Hefnawy, Tribology International, 17, 3 (1984) 